\title{
Asymmetric electroosmotic pumping across porous media sandwiched with perforated ion-exchange membranes
}

\author{
A. Yaroshchuk, (DD *ab E. E. Licón, ${ }^{\text {b }}$ E. K. Zholkovskiy, ${ }^{c}$ M. P. Bondarenko ${ }^{c}$ \\ and T. Heldal ${ }^{\text {d }}$
}

Received 5th December 2016, Accepted 25th January 2017

DOI: $10.1039 / c 6 f d 00248 j$

To have non-zero net flow in AC electroosmotic pumps, the electroosmosis (EO) has to be non-linear and asymmetric. This can be achieved due to ionic concentration polarization. This is known to occur close to micro-/nano-interfaces provided that the sizes of the nanopores are not too large compared to the Debye screening length. However, operation of the corresponding EO pumps can be quite sensitive to the solution concentration and, thus, unstable in practical applications. Concentration polarization of ion-exchange membranes is much more robust. However, the hydraulic permeability of the membrane is very low, which makes EO flows through them extremely small. This communication shows theoretically how this problem can be resolved via making scarce microscopic perforations in an ion-exchange membrane and putting it in series with an EO-active nano-porous medium. The problem of coupled flow, concentration and electrostatic-potential distributions is solved numerically by using finite-element methods. This analysis reveals that even quite scarce perforations of micron-scale diameters are sufficient to observe practically-interesting EO flows in the system. If the average distance between the perforations is smaller than the thickness of the EOactive layer, there is an effective homogenization of the electrolyte concentration and hydrostatic pressure in the lateral direction at some distance from the interface. The simulations show this distance to be somewhat lower than the half-distance between the perforations. On the other hand, when the surface fraction of perforations is sufficiently small (below a fraction of a percent) this "homogeneous" concentration is considerably reduced (or increased, depending on the current direction), which makes the EO strongly non-linear and asymmetric. This analysis provides initial guidance for the design of high-productivity and inexpensive AC electroosmotic pumps.

aICREA, pg. L. Companys 23, 08010 Barcelona, Spain

${ }^{b}$ Dept of Chemical Engineering, Polytechnic University of Catalonia, Av. Diagonal 647, 08028 Barcelona, Spain. E-mail: andriy.yaroshchuk@upc.edu

'Institute of Bio-Colloid Chemistry, National Academy of Sciences of Ukraine, Vernadskiy Ave. 42, 03142, Kyiv, Ukraine

${ }^{d}$ Osmotex AG, Schützenstrasse 3, 8800 Thalwil, Switzerland 


\section{Introduction}

Electro-osmotic pumps are useful because they have no moving parts and can be easily miniaturized for applications in micro-Total-Analysis Systems ( $\mu \mathrm{TAS}){ }^{1}$ Another interesting application is EO-generated "active" moisture removal through engineered sports tissues., ${ }^{2,3}$

Alternating Current EO pumps (ACEO) have the potential advantage of exhibiting no electrode reactions (provided that the voltage is not too high and/or the frequency is not too low). The absence of electrode reactions is a sine-qua-non in the application of "active" moisture removal because of the direct contact of the material with the human body.

For an ACEO pump to produce net flows (over the AC period), the EO has to be asymmetric (its rate is dependent on the current direction). Over the last decade, considerable efforts have been devoted to the exploration of ACEO based on the concept of travelling-wave ACEO using asymmetric electrode arrays. ${ }^{4-10}$

To be asymmetric, the EO must be non-linear. One of the mechanisms of EO non-linearity is due to ionic concentration polarization (CP). Recently, this has been studied extensively both theoretically and experimentally in micro-/nanofluidic systems (see, for example, this review ${ }^{\mathbf{1 1}}$ ). The mechanism of CP-induced EO non-linearity is simple. In sufficiently fine-porous media (or narrow nanochannels), the ion transport numbers are noticeably different from the electrolyte solution (due to the existence of EDLs whose diffuse parts make up a nonnegligible portion of pore space). Therefore, current passage gives rise to changes in electrolyte concentration at and around the interfaces between the media and electrolyte solutions. The rate of electroosmosis increases with decreasing concentration. Due to the concentration polarization, salt concentration either decreases or increases close to the interfaces depending on the current direction and sign of the surface charge. This can give rise to non-linear EO. Additionally, if the system is asymmetric, the rate of non-linear EO in one current direction can be different from its rate in the other. As a result, there can be net EO flows in response to alternating currents with zero net electric charge transfer.

The required asymmetry occurs, for example, in nano-funnels, ${ }^{12}$ or in tracketched membranes with conical nano-pores, ${ }^{13-15}$ and, indeed, net ACEO pumping has been demonstrated experimentally for such systems. However, for the concentration polarization to be sufficiently strong, the smallest dimension of the nano-channel cannot be too large compared to the Debye screening length, otherwise the concentration polarization is "suppressed" by electroosmosis itself. ${ }^{16}$ This can easily make the system operation unstable in practical applications. Additionally, in the context of nano-engineered devices, there are issues of low productivity and extremely high cost.

In this communication, we will show how the concentration-polarization phenomena can be largely "decoupled" from the (nano)-porous medium but still influence the electroosmosis through it in the desired way. This can be achieved by using a novel micro-engineered layered material.

Current-induced concentration polarization is well-known to occur close to the surfaces of ion-exchange membranes, and it is actually the physico-chemical mechanism of electrodialysis. ${ }^{17}$ Ion-exchange membranes are usually polymeric 
materials with considerable concentrations of dissociable groups being a part of (or being covalently attached to) the polymer backbone. In water (and/or other polar solvents) these groups dissociate and give rise to immobile (fixed) electric charges. Due to the electroneutrality requirement, their charge is compensated by mobile counterions whose concentration in the membrane phase can be considerably higher than in the equilibrium solution. Conversely, the concentration of ions of opposite charge (co-ions) can be considerably reduced. This can give rise to a pronounced asymmetry in the permeability of ion-exchange membranes to counterions and co-ions, thus making the conductance of ionexchange membranes practically unipolar. Due to the very small pore size and high ion-exchange capacity (fixed-charge concentration) of typical ion-exchange membranes, this can occur even up to quite high salinities. Therefore, if an EO-active porous medium is in direct contact with an ion-exchange membrane, the salt concentration at the interface between them decreases or increases (depending on the current direction) even in solutions of quite high salinity. The pore size of the EO-active medium is not so crucial in this case (in contrast to single nano-porous media where both the CP and EO are controlled by the same material). This layered arrangement would potentially make the EO strongly nonlinear and asymmetric, provided that the ion-exchange membrane is on one side of the EO-active medium only. However, the hydraulic permeability of conventional ion-exchange membranes is extremely low. Therefore, with them, the EOactive medium would be working in the mode of EO pressure where gradients of hydrostatic pressure arise inside the "sandwich" but the volume flow through it is negligible.

The idea is to considerably increase the hydraulic permeability of the ionexchange layer via making microscopic pores/channels in it. This arrangement is schematically depicted in Fig. 1.

If the average distance between the openings is essentially smaller than the thickness of the EO-active layer, one can expect effective homogenization of electrolyte concentration in the lateral direction at some distance from the interface. At the same time, when the surface fraction of "perforations" is sufficiently low, the concentration-polarization phenomena may not be critically affected by the presence of the "openings". To be more precise (as shown below), the electroosmosis itself does noticeably affect the concentration polarization (and, for example, makes the limiting current disappear), but the EO remains a strongly non-linear and asymmetric function of current density, nonetheless.

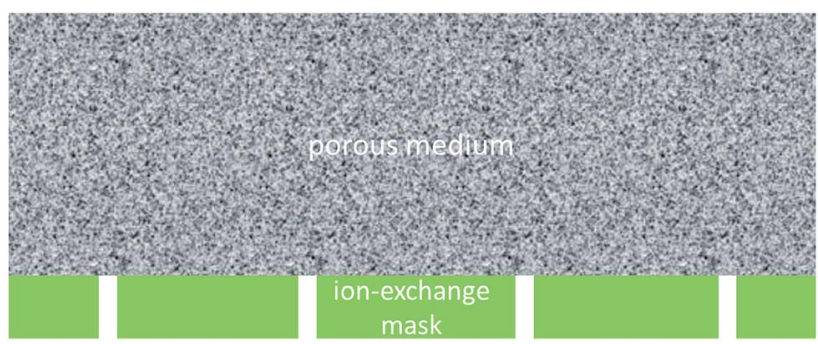

Fig. 1 Sketch of a nano-porous layer sandwiched with a perforated ion-exchange mask (not to scale). 
We will investigate this system theoretically via analysis of the limiting cases and numerical simulations and show that this physical picture is correct.

\section{Theory}

\section{Problem formulation}

To our knowledge, EO in the system of interest has never been studied before. Therefore, we will use the simplest non-trivial problem formulation. We will consider a symmetrical $(1: 1)$ salt with ions of equal mobilities, we will neglect streaming current, and we will describe the EO according to the Smoluchowski model (no surface conductance and no Electrical Double Layer (EDL) overlap). We will also consider the zeta-potential constant (independent of salt concentration). Most of these approximations are legitimate if the pores of the EO-active medium are much larger than the Debye screening length. The latter is about $3 \mathrm{~nm}$ in length in practically-relevant centi-molar solutions (10 $\mathrm{mM})$. Thus, if the pore size is much larger than that (for example, $200 \mathrm{~nm}$ as in the simulations below) the overlap of EDLs can be clearly neglected. Besides, if the pore-surface (zeta) potential is not too high, one can also neglect the additional conductance due to the counterions that are attracted to the pore surface (surface conductance) as compared to the conductance in the bulk of the pore volume. The streaming contribution to the current is also proportional to the fraction of the pore volume occupied by the EDLs, and can be neglected for the same reason.

The assumption of a constant zeta-potential is unrelated to the pore size and somewhat artificial because usually the zeta-potential increases in absolute value with decreasing concentration. However, this approximation makes possible the decoupling of the hydraulic problem, which helps a lot with elucidating the physics. At the same time, from the analysis below, it will be seen that disregarding the possible increase in the absolute value of the zeta-potential with decreasing salt concentration most probably leads to underestimating the EO asymmetry, therefore with a more realistic model the effect can probably only be stronger. This will be investigated in future studies.

In the above approximations, for the electric-current density, $\vec{I}$, volume flux, $\vec{J}_{\mathrm{v}}$, and salt flux, $\vec{J}_{\mathrm{s}}$, we can write down these constitutive relationships

$$
\begin{aligned}
\vec{I}=-\alpha \cdot c \cdot \vec{\nabla} \varphi & (\text { Ohm's law }) \\
\vec{J}_{\mathrm{v}}=-\chi \cdot \vec{\nabla} P+\beta \cdot \vec{\nabla} \varphi & \text { (extended Darcy law) } \\
\vec{J}_{\mathrm{s}}=-D \cdot \vec{\nabla} c+c \cdot \vec{J}_{\mathrm{v}} & \text { (convection-diffusion) }
\end{aligned}
$$

where

$$
\begin{gathered}
\alpha \equiv \frac{2 F^{2} D \gamma}{R T} \\
\beta \equiv \frac{\varepsilon \varepsilon_{0} \zeta \gamma}{\eta}
\end{gathered}
$$

$c$ is the salt concentration, $\varphi$ is the electrostatic potential, $P$ is hydrostatic pressure, $\chi$ is the mechanical permeability of the porous medium, $D$ is the salt diffusion coefficient, $\varepsilon \varepsilon_{0}$ is the dielectric constant, $\zeta$ is the zeta-potential of the 
pore surface, $\eta$ is solution viscosity, and $\gamma$ is the porous-medium effective porosity (accounting for the pore tortuosity). The extended Darcy law states that the volume flow is a superposition of electroosmosis and pressure-driven flow.

The electric-current density, volume flux and salt flux must be conservative

$$
\begin{gathered}
\operatorname{div}(\vec{I})=0 \\
\operatorname{div}\left(\vec{J}_{\mathrm{v}}\right)=0 \\
\operatorname{div}\left(\vec{J}_{\mathrm{s}}\right)=0
\end{gathered}
$$

and by substituting eqn (1)-(3) to (6)-(8) we obtain

$$
\begin{gathered}
c \cdot \nabla^{2} \varphi+(\vec{\nabla} c \cdot \vec{\nabla} \varphi)=0 \\
\beta \cdot \nabla^{2} \varphi-\chi \cdot \nabla^{2} P=0 \\
-D \cdot \nabla^{2} c-\chi \cdot(\vec{\nabla} c \cdot \vec{\nabla} P)+\beta \cdot(\vec{\nabla} c \cdot \vec{\nabla} \varphi)=0
\end{gathered}
$$

To model the ion-exchange layer, in the first approximation we consider an infinitely thin but hydraulically impermeable and ideally perm-selective ionexchange film with negligible ohmic resistance. The latter assumptions correspond to the limiting case of very large fixed-charge densities. In such a hydraulic "mask", there are openings (holes) where the surface of the porous medium is exposed (uncovered). In this study, we consider a layered system surrounded by sufficiently large volumes of solution so that the pressure gradients are localized exclusively inside the system. The external hydrostatic-pressure difference between the solutions separated by the "sandwich" is zero (classical conditions of EO) and the zero pressure condition is supposed to extend to the system's external surfaces. The salt concentration is the same in both solutions and extends right up to the system's surfaces (external concentration polarization is disregarded). The electrostatic potential is also assumed to be set immediately at the system's surfaces, but there is a potential difference between the solutions separated by the

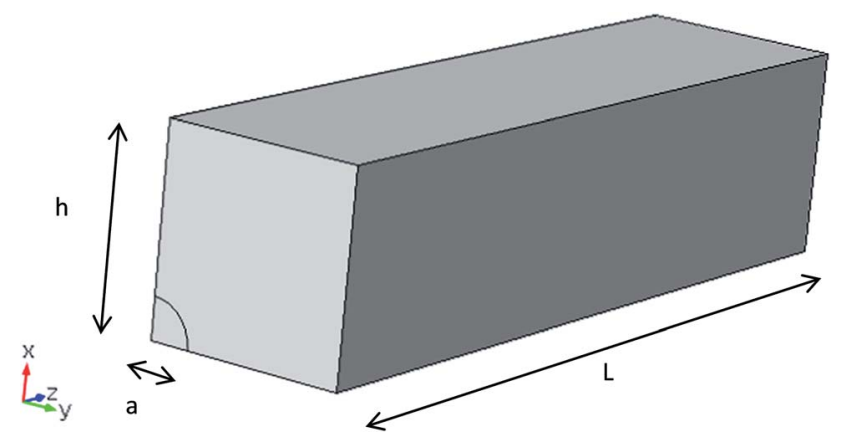

Fig. 2 Computational domain, coordinates and dimensions. 
system (applied voltage). This is the only external driving force. The system and the coordinates are schematically shown in Fig. 2.

Accordingly, the boundary conditions are:

At the hole surface ("lower" solution reservoir)

$$
\begin{gathered}
c=c_{0} \\
P=0 \\
\varphi=\varphi_{0}
\end{gathered}
$$

At the opposite porous-medium surface ("upper" solution reservoir)

$$
\begin{aligned}
& c=c_{0} \\
& P=0 \\
& \varphi=0
\end{aligned}
$$

At the mask/porous-medium interface ("lower" side) we use the conditions of hydraulic impermeability of the mask

$$
\left.\vec{J}_{\mathrm{v}}\right|_{n}=\left.0 \quad \vec{\nabla} P\right|_{n}=\left.\frac{\beta}{\chi} \cdot \vec{\nabla} \varphi\right|_{n}
$$

of zero flux of co-ions (ideal mask perm-selectivity)

$$
\left.\vec{\nabla} c\right|_{n}+\left.\frac{F}{R T} \cdot Z_{\mathrm{c}} \cdot c \cdot \vec{\nabla} \varphi\right|_{n}=0
$$

and of zero difference of the electrochemical potential of counter-ions across the mask (negligible ohmic resistance)

$$
\ln \left(\frac{c}{c_{0}}\right)+\frac{F}{R T} \cdot Z_{\mathrm{g}} \cdot\left(\varphi-\varphi_{0}\right)=0
$$

$Z_{\mathrm{g}}$ and $Z_{\mathrm{c}}$ are the charges of the counter-ions and co-ions and they are defined for the ion-exchange mask. In this study we consider the ion-exchange layer to be anion-perm-selective (positive fixed charge) and the EO-active layer to have a negative fixed charge.

In view of the system symmetry, at the external cell boundary we set equal to zero the normal derivatives of all the sought-after quantities, namely, the electrostatic potential, hydrostatic pressure and salt concentration. The geometry of this boundary depends on the shape and location of the holes. In this study we consider square lattices of circular holes. Accordingly, the external cell boundary is the surface of a box with a square cross-section as shown in Fig. 2.

\section{Solution procedure}

Decoupling of the hydraulic problem. Inspection of the equations and boundary conditions reveals that in this formulation the hydraulic problem (description of the volume flow) can be decoupled from the electrochemical problem (description of the electrostatic-potential and salt-concentration distributions). By using eqn (10) we can introduce an effective pressure according to 


$$
A \equiv P-\frac{\beta}{\chi} \cdot \varphi
$$

The volume flow is proportional to the negative gradient of this function. Due to the volume conservation, it satisfies the Laplace equation. A separate set of necessary and sufficient boundary conditions can be formulated for this problem too,

$$
\begin{gathered}
A=-\frac{\beta}{\chi} \cdot \varphi_{0} \text { at the hole surface } \\
A=0 \text { at the back (upper) medium surface } \\
\left.\vec{\nabla} A\right|_{n}=0 \text { at the mask/medium interface }
\end{gathered}
$$

Remarkably, this is a linear problem, so both the effective pressure and volume flow are just proportional to the applied voltage, $\varphi_{0}$. Moreover, the solution of the hydraulic problem is not dependent on the distribution of salt concentration. It also does not explicitly depend on the distribution of the electrostatic potential (although this has an indirect impact via the effective pressure). Therefore, the hydraulic problem can be solved separately. Due to the linearity in the applied voltage, for a given system geometry this has to be done only once. After that, the electrochemical problem should be solved with a given flow distribution. This is a non-linear problem, which gives rise to non-linear current-voltage characteristics and ultimately to the non-linear dependence of the EO rate on current density.

Numerical procedure. Numerical simulations were carried out using Comsol Multiphysics software and the finite element method (FEM). A free triangular mesh was generated with a refinement at the edge of the hole with a minimum element size of $0.1 \mu \mathrm{m}$. The minimum element size was set to $0.5 \mu \mathrm{m}$ in the vicinity of the hole until the distance equal to the cell size $(2 h)$ in the $z$-direction was reached. After that, the minimum element size was increased to $10 \mu \mathrm{m}$. The system of PDEs was solved by the Multifrontal Massively Parallel Sparse direct Solver (MUMPS) with a relative tolerance of 0.001 , using a CELSIUS workstation (Fujitsu) with an Intel ${ }^{\circledR}$ Xeon ${ }^{\circledR}$ Processor E5-2620 v2 (15M Cache, $\left.2.10 \mathrm{GHz}\right)$ and $48 \mathrm{~GB}$ of RAM.

Table 1 Fixed parameters used in the numerical simulations

\begin{tabular}{ll}
\hline Parameter & Value \\
\hline Porous-layer thickness, $L$ & $100 \mu \mathrm{m}$ \\
Porous-layer pore radius & $100 \mathrm{~nm}$ \\
Active porosity, $\gamma$ & 0.5 \\
Solution viscosity, $\eta$ & $0.001 \mathrm{~Pa} \mathrm{~s}$ \\
Relative dielectric permittivity, $\varepsilon$ & 80 \\
Zeta potential, $\zeta$ & $-0.03 \mathrm{~V}$ \\
Diffusion coefficient, $D$ & $2 \times 10^{-9} \mathrm{~m}^{2} \mathrm{~s}^{-1}$ \\
Temperature, $T$ & $298 \mathrm{~K}$ \\
Outside concentration, $c_{0}$ & $30 \mathrm{mM}$
\end{tabular}


The values of some parameters were fixed and they are listed in Table 1 . The selected value for the zeta-potential $(-30 \mathrm{mV})$ is typical for polymeric surfaces in (1:1) electrolyte solutions of moderate concentrations. ${ }^{18}$ The diffusion coefficient is that of $\mathrm{KCl}$. The concentration of $30 \mathrm{mM}$ is typical for human "external physiological fluids" (the application of active moisture removal). The hydraulic permeability of the porous medium was calculated by using the Hagen-Poiseuille relationship for straight cylindrical capillaries.

Within the scope of our simplified problem formulation, the impact of some of these parameters (salt concentration, salt diffusion coefficient, porous-medium active porosity and pore size, and fluid viscosity) is rather trivial because their variation leads only to a rescaling of the current density or hydrostatic-pressure magnitude. The effect of other parameters (porous-medium thickness and zetapotential) is less trivial and deserves a more detailed study. The hole shape and location can also have some non-trivial effects. However, the purpose of this communication is to demonstrate the principal features of the system. A more complete parametric study will be published elsewhere.

\section{Results and discussion}

\section{Hydraulic problem}

Limiting cases of no mask and continuous mask (no holes). We start by briefly recapitulating these two well-known reference cases.

No mask. The hydrostatic pressure inside the porous medium is zero (classical EO mode) and the effective pressure is just proportional to the electrostatic potential.

$$
A \equiv-\frac{\beta}{\chi} \cdot \varphi
$$

Continuous mask (no holes). The effective pressure is zero inside the porous medium (classical EO-pressure mode)

$$
A=0
$$

and the volume flow is zero, too.

Limiting case of non-interacting holes and an infinitely thick porous medium. In this sub-section, we consider the limiting case of a single hole and an infinitelythick porous medium where there is an analytical solution. This solution will be used to obtain a simple scaling law for the dependence of EO flow on hole size and inter-hole distance when the size is small enough and the distance is sufficiently large.

Eqn (10) and (22)-(24) are formally equivalent to the problem of the distribution of electrostatic potential around a conducting infinitely thin charged circular disk in a vacuum. Instead of the semi-infinite space, we should consider an infinite space so that the condition of eqn (24) follows from the symmetry with respect to the plane of the disk. The solution of such an electrostatic problem can be found in textbooks (see, for example, ref. 19). Being proportional to the normal derivative of the electrostatic potential, the surface charge density in the electrostatic problem is analogous to the normal derivative of the effective pressure in 
the hydraulic problem. The electrostatic potential itself is analogous to the effective pressure.

With this analogy in mind, we can write down this expression for the distribution of effective pressure

$$
A(r, z)=-\frac{2 \beta \varphi_{0}}{\pi a \chi} \cdot \operatorname{atan}\left(\frac{\sqrt{2} a}{\sqrt{r^{2}+z^{2}-a^{2}+\sqrt{\left(r^{2}+z^{2}-a^{2}\right)^{2}+4 a^{2} z^{2}}}}\right)
$$

where $a$ is the hole radius. For the normal volume flux at the hole surface, we obtain

$$
\left.J_{\mathrm{v}}\right|_{z}(r)=\frac{Q}{2 \pi a^{2}} \cdot \frac{1}{\sqrt{1-\left(\frac{r}{a}\right)^{2}}}
$$

where

$$
\left.Q \equiv 2 \pi \cdot \int_{0}^{a} \mathrm{~d} r r \cdot J_{\mathrm{v}}\right|_{z}(r)
$$

is the volumetric flow (in $\mathrm{m}^{3} \mathrm{~s}^{-1}$ ) through the hole. It can be related to the electrostatic potential (for the hydraulic problem) at the hole surface in this simple way

$$
Q=-4 \beta \cdot a \cdot \varphi_{0}
$$

Remarkably, this volumetric flow is proportional to the hole radius and not to its area as one might expect.

Now, we should take into account that there are actually other holes, but we consider the volume transfer through them as independent from each other (and, thus, the total flux is just the superposition of flows through single holes). One can define a per-hole surface area, $S$, and consider the case of no mask as a reference. In this latter case, the distribution of electrostatic potential in the porous medium is $1 \mathrm{D}$ and linear, so for the EO volumetric flow through the surface area of $S$ we obtain

$$
Q_{0}=-\beta \cdot S \cdot \frac{\varphi_{0}}{L}
$$

where $L$ is the porous-medium thickness. Finally, we can define a hydraulic shielding factor, $\theta$, as the ratio of volumetric flow with the mask and without the mask. From eqn (30) and (31), we can see that in the limit of non-interacting holes

$$
\theta \equiv \frac{Q}{Q_{0}}=\frac{4 a L}{S} \equiv \frac{a L}{h^{2}}
$$

Fig. 3 shows the shielding factor $v s$. the hole radius for several inter-hole halfdistances.

Indeed, the shielding factor scales linearly with the radius when the latter is sufficiently small. As was expected, the larger the inter-hole spacing the broader the scope of applicability of non-interacting holes. Rather less expectedly, the 


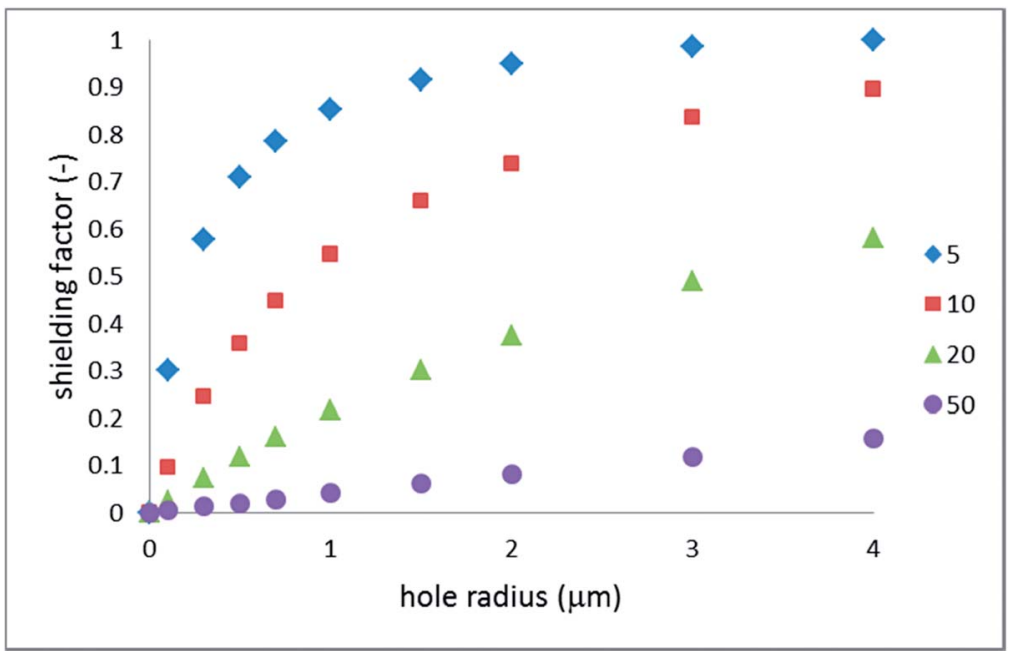

Fig. 3 Hydraulic shielding factor vs. hole radius; the legend indicates the cell size (halfdistance between the hole centers) in micrometers.

shielding factor is close to one (weak shielding) even for relatively small surface fractions of the holes.

Fig. 4 provides additional insight into the applicability of non-interacting holes. Fig. 4a shows the shielding factor calculated numerically along with the scaling law of eqn (32). Fig. $4 \mathrm{~b}$ shows the relative error of this approximation.

There is a range where the approximation works quite well (the inter-hole spacing above $40 \mu \mathrm{m})$. At the same time, at smaller spacing the accuracy quickly deteriorates, hence the holes interact considerably. Noticeably, this occurs already at the inter-hole spacing, which is 15 times larger than the hole diameter, for example. One can also see that the relative error does not tend to zero with increasing cell size but appears to approach a saturation value of about $-10 \%$. This is probably due to the finite thickness of the porous medium which is assumed in the numerical simulations, whereas the analytical solution considers this medium as infinitely thick. There is also a change of sign of the relative error. A positive error means that the approximation overestimates the flow. At smaller inter-hole spacings, the per-hole flow is reduced due to the inter-hole interaction

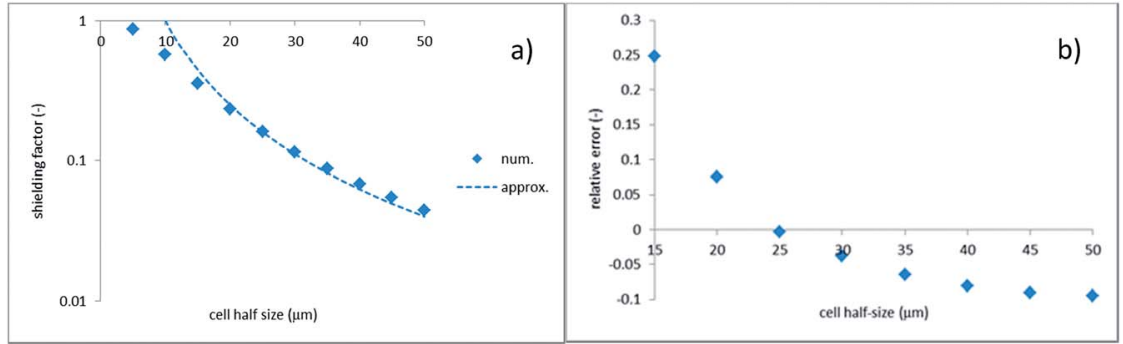

Fig. 4 Shielding factor vs. half inter-hole spacing; hole radius is $1 \mu \mathrm{m}$; (a) numerics vs. scaling law of eqn (32); (b) relative error of scaling law. 
(see below). At larger spacings, the dominant factor is the finite porous-medium thickness, which makes the flow somewhat larger compared to the analytical solution.

Eqn (27) shows that at distances exceeding the hole size, the effective pressure is distributed in an approximately semi-spherical way. In the electrostatic analogy, the effective pressure is analogous to the electrostatic potential of a charged disk. When sufficiently far away from it, the potential distribution approaches that of a point-like source. The electrostatic analogy also reveals that the holes behave as non-interacting as long as they can be considered as pointlike (i.e., the inter-hole spacing is much larger than the hole size). Indeed, the electrostatic potentials of point-like sources just superimpose. Interactions between the holes can also be understood in electrostatic terms. If charged conducting disks (being at the same electrostatic potential) approach each other, the charges on them redistribute. This gives rise to some reduction of integral charges (analogous to the per-hole volumetric flows). Besides, there is a redistribution of charge density (analogous to the local fluid velocity normal to the hole surface). In electrostatic terms, this results in the appearance of multipole moments. For the square-lattice hole geometry considered in this study, the dipole moment is zero due to the symmetry. The first non-zero moment is quadrupole.

The analysis of the shielding factor is important because it quantifies to what extent the EO flow is reduced due to the impermeable mask. This reduction is superimposed with the increase in the voltage drop (at a given current density) due to the concentration polarization.

\section{Electrochemical problem}

Numerical results. The electrochemical problem is a system of two PDEs for the salt concentration and electrostatic potential. Remarkably, once the flow field is found from the solution of the decoupled hydraulic problem, the convectiondiffusion equation of eqn (11) does not explicitly contain the electrostatic potential. However, it is coupled to the potential distribution via the boundary conditions of eqn (19) and (20). The system of the PDE (along with the separate PDE for the hydraulic problem) was solved numerically by using Comsol Multiphysics software as described above.

Concentration distributions. Fig. 5 shows some examples of salt-concentration distributions.

These color maps reveal several important features. Firstly, at distances (in the vertical direction) exceeding the inter-hole spacing, the concentration distribution is perfectly homogeneous in the horizontal plane. Of course, color maps do not really provide quantitative insight but below we will support this statement with conventional graphs. Secondly, at intermediate distances from the hole, the concentration-distribution field is quasi-semi-spherical. Very close to the hole, the distribution is flattened due to the constant concentration set at the hole surface. Both these features are similar to the distribution of effective pressure in the hydraulic problem. Thirdly, with increasing voltage, the 1D concentration distribution in the vertical direction becomes more non-linear.

Fig. 6 shows concentration profiles along the horizontal axis (calculated where the cutline passes through the hole center). 

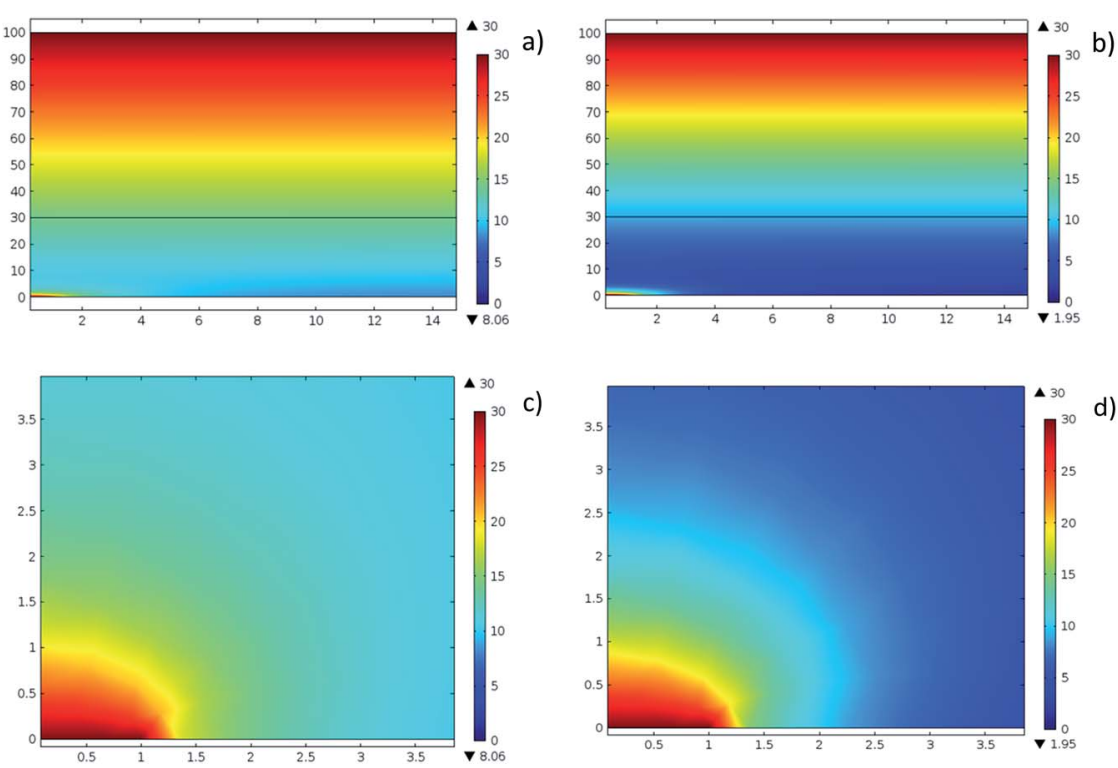

b)

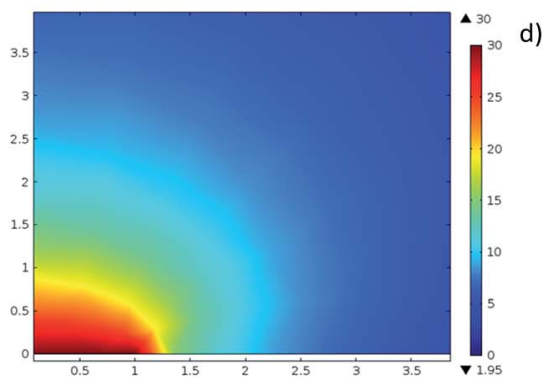

Fig. 5 Salt-concentration distributions in the vertical plane passing through the hole center: hole radius $1 \mathrm{m \mu}$, cell half-size $15 \mu \mathrm{m}$; in the upper maps $(a, b)$ the scales along the vertical and horizontal axes are different; the lower maps (c, d) show the magnified vicinity of the hole with the same scaling along both axes. The hole center is in the lower left corner. (a, c) $\varphi_{0}=93 \mathrm{mV}$; (b, d) $\varphi_{0}=200 \mathrm{mV}$.

The inter-hole spacing in this simulation was $30 \mu \mathrm{m}$. Nonetheless, one can see that, even at a distance of as little as $6.8 \mu \mathrm{m}$ from the mask/porous-medium interface, the concentration varies in the horizontal direction by only about

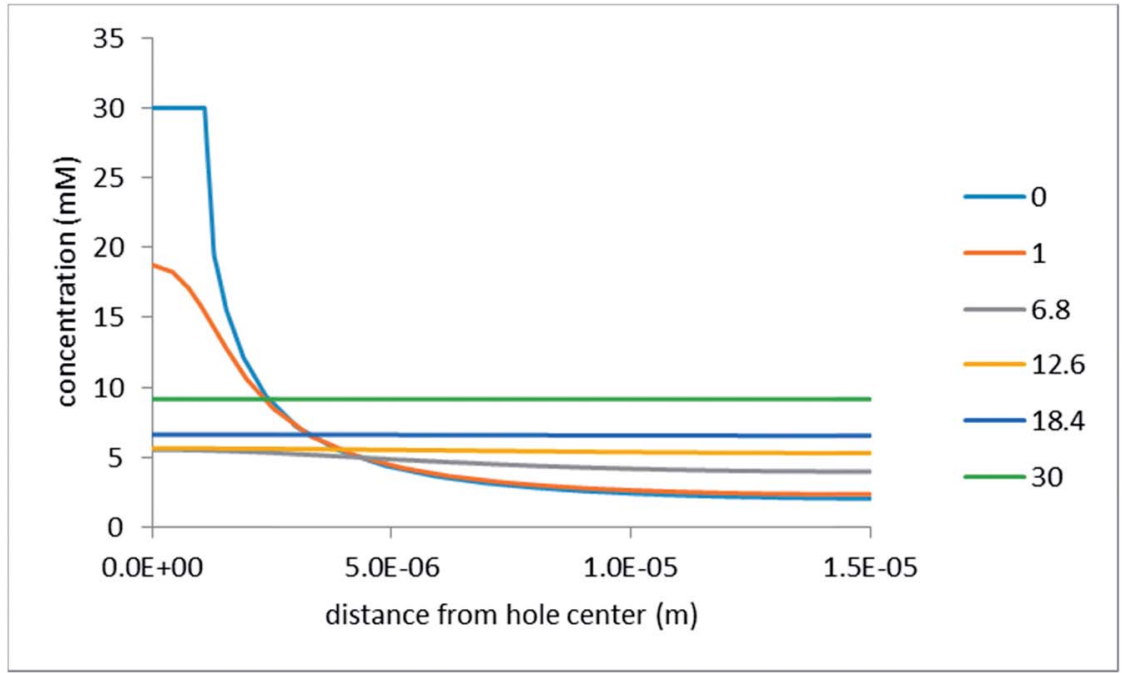

Fig. 6 Salt-concentration profiles in the horizontal direction at various distances (in $\mu \mathrm{m}$ ) from the mask/porous-medium interface as indicated in the legend: hole radius $1 \mu \mathrm{m}$, inter-hole spacing $30 \mu \mathrm{m}$, voltage drop $200 \mathrm{mV}$. 
$25 \%$. At a distance of $12.6 \mu \mathrm{m}$ (still more than 2 times smaller than the inter-hole spacing) the concentration remains practically constant. Notably, the voltage drop in the simulations shown in Fig. 6 was assumed to be quite high $(200 \mathrm{mV})$. At lower voltages, the concentration distribution in the horizontal direction is even more homogeneous (at some distance from the interface). At the same time, very close to the interface, the concentration distribution is strongly inhomogeneous. The same is characteristic of the distributions of the electrostatic potential and effective pressure.

Fig. 7 illustrates the non-linearity of the concentration profiles in the vertical direction.

Indeed, with increasing voltage the profiles become ever more concave. This is more pronounced for the smaller inter-hole spacings because the non-linearity is caused by the volume flow, which is larger in this case. This is additionally highlighted by Fig. 7c, which compares several inter-hole spacings at the same voltage. Along with the changes in the extent of non-linearity, one can see that, as is expected, the overall reduction in the concentration is stronger at larger inter-hole spacings.

Fig. 7 shows the concentration profiles calculated for the $z$-cutline, which is the most distant from the hole center. There, the concentration reduction is most pronounced. For comparison, Fig. 8 shows concentration profiles for a $z$-cutline passing through the hole edge.

As discussed above (see the section on the hydraulic problem), here the rate of vertical volume inflow is the largest. One can see that despite this, the concentration drops abruptly (probably due to the diffusor nature of the flow in this case), passes through a minimum, and after that crosses over to the largely $1 \mathrm{D}$ behavior typical at larger distances from the interface (this can be clearly seen from a comparison with Fig. 7c).
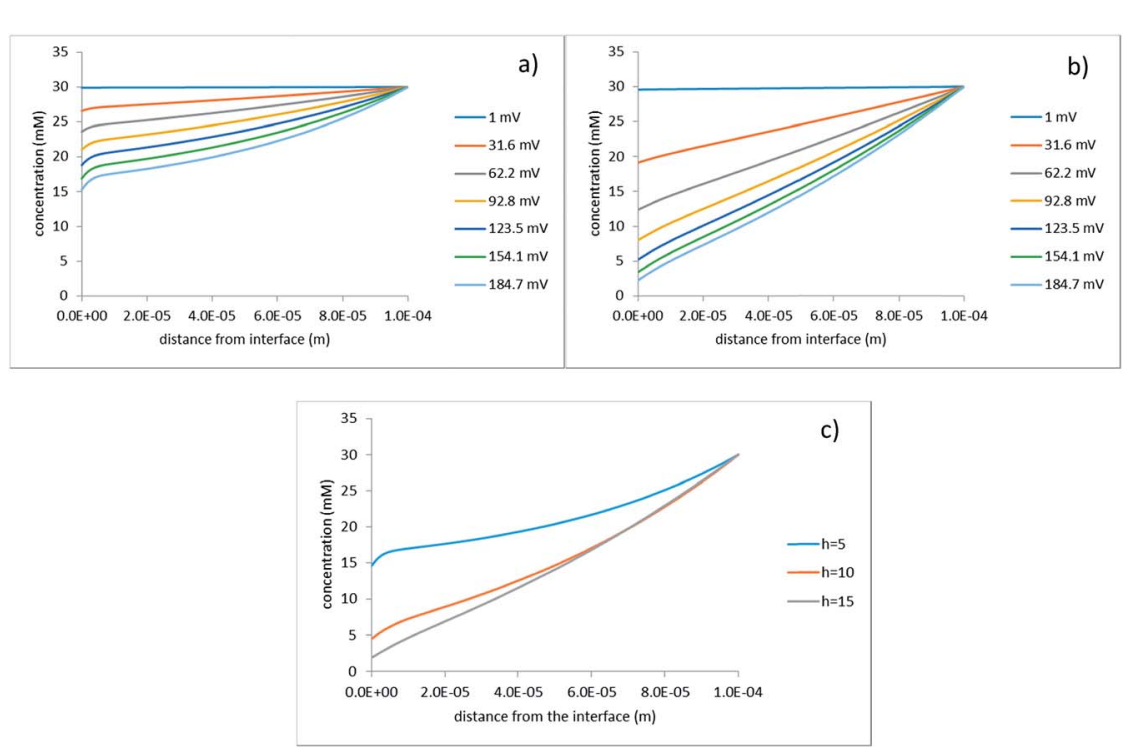

Fig. 7 Concentration profiles along the $z$-axis at the upper right cell corner (see Fig. 2); (a) $h=5 \mu \mathrm{m}$; (b) $h=15 \mu \mathrm{m}$; the legends indicate the applied voltage; (c) $\varphi_{0}=200 \mathrm{mV}$, the legend indicates the half-distance between the hole centers in micrometers. 


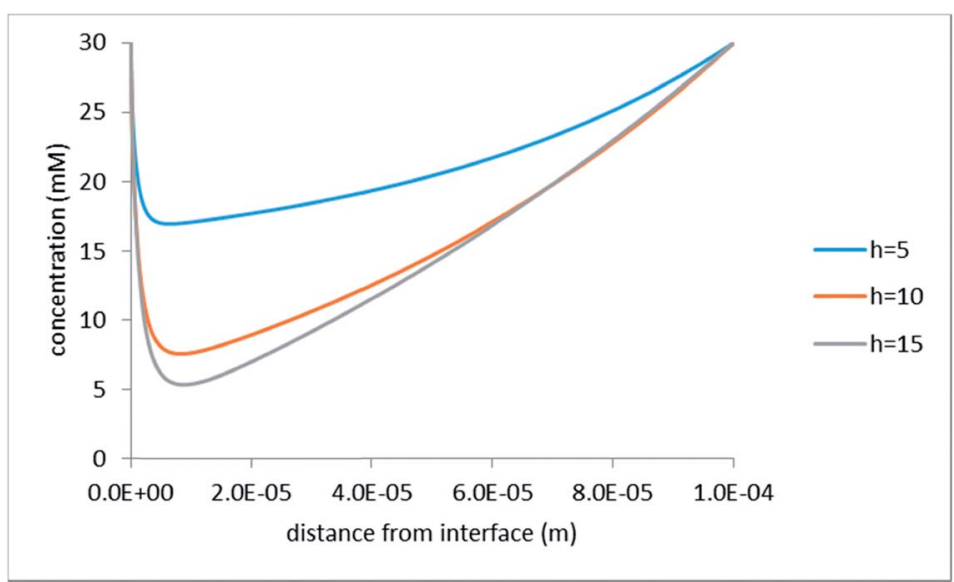

Fig. 8 Concentration distribution along the $z$-axis at the hole edge; $\varphi_{0}=200 \mathrm{mV}$; the legend indicates half-distance between the hole centers in micrometers.

In the context of ACEO, we are also interested in negative voltages because ultimately, we need to obtain the net EO flow over the period of AC. In this steadystate analysis, we imply that the frequency of AC is sufficiently low for the system to always be in a quasi-steady state. The net EO flow over the AC period is then the algebraic sum of the volume flows occurring at current densities of the same magnitude but with opposite signs. Fig. 9 shows the concentration profiles calculated for a set of negative voltages.

As is expected, in this case the concentration at the interface increases, which becomes ever more pronounced with growing voltage. This increase in concentration gives rise to larger current densities and super-linear current-voltage characteristics as discussed below.

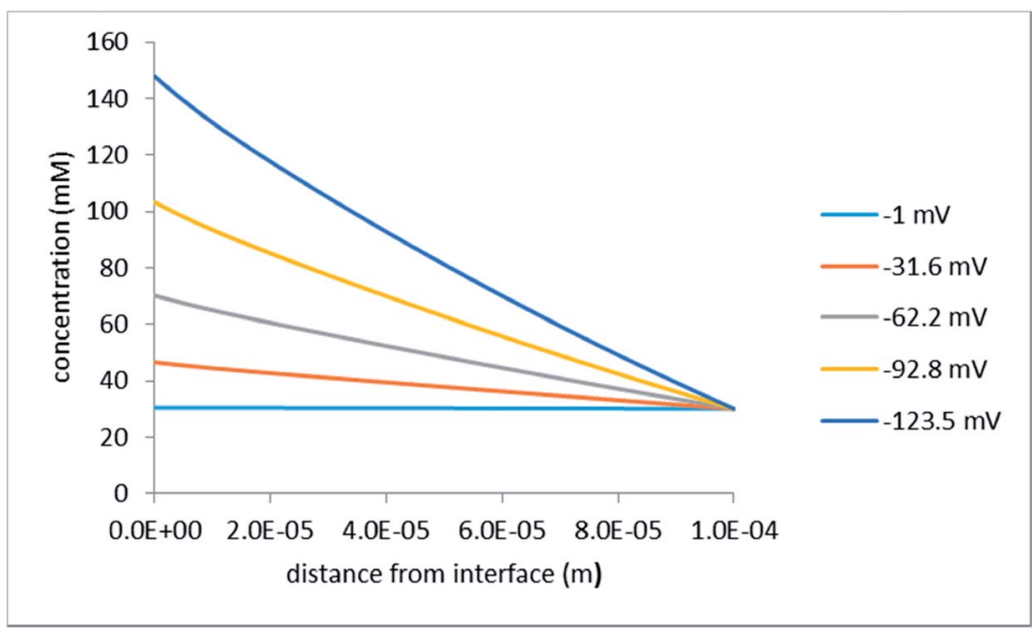

Fig. 9 Concentration distribution along the $z$-axis at the upper right cell corner (see Fig. 2); the legend indicates the applied voltages; the half-distance between the hole centers is $15 \mu \mathrm{m}$. 
Current-voltage characteristics. In view of the strongly reduced salt concentration (at positive voltages) being a pronounced function of the applied voltage, one can expect strongly non-linear current-voltage characteristics. This is confirmed by Fig. 10. In a typical diode-like manner, one part is sublinear while the other is super-linear. Along with the results of the numerical simulations, Fig. 10 shows the limiting cases of no mask and no holes where simple analytical solutions are available (see below).

For the no mask example, the current-voltage characteristics is just linear

$$
I=\frac{2 F^{2} \cdot D \gamma \cdot c_{0} \cdot \varphi_{0}}{R T \cdot L}
$$

For the no holes (continuous mask) example, one can obtain this relationship

$$
I=\frac{2 F \cdot D \gamma \cdot c_{0}}{L} \cdot\left(1-\exp \left(-F \varphi_{0} / 2 R T\right)\right)
$$

This case can be solved in this simple way because we neglect the ohmic voltage drop on the ion-exchange layer (otherwise one would obtain a transcendental equation for the current density).

Remarkably, the initial slope of the current-voltage characteristics for the no holes case is two times smaller than the slope of the globally linear characteristics for the no mask case. This occurs because (in our idealized system with an ideally perm-selective and conducting mask) exactly $50 \%$ of the applied voltage arises on the mask as concentration potential.

Fig. 10 shows that at positive voltages all the curves calculated for the perforated masks are confined between those two limiting cases. At negative voltages, the situation is somewhat more complex. When the voltage is low, the picture is dominated by the concentration potential arising on the mask, which

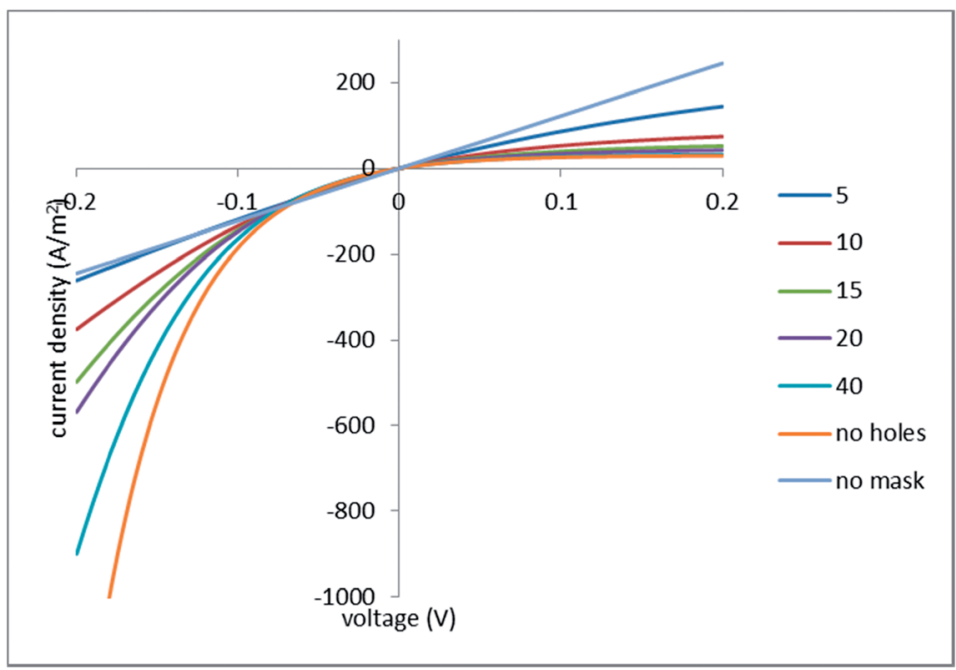

Fig. 10 Current-voltage characteristics; the legend indicates the half-spacing between the holes (in micrometers); the hole radius is $1 \mu \mathrm{m}$. 
(similarly to the positive voltages) has the same sign as the applied voltage. As a result, all the curves calculated for the perforated masks (including the limiting case of no holes) run above the limiting case of no mask (that is, the absolute values of current density are smaller). At larger voltages, a decrease in the ohmic resistance of the porous medium, due to the increase in the concentration, takes over, and the "masked" cases exhibit larger absolute values of current density.

Flow-voltage characteristics. Fig. 11 shows volume-flow rate vs. applied voltage. These dependencies are strictly linear, which is in agreement with the decoupling of the hydraulic problem as discussed above. Increasing the inter-hole spacing gives rise to decreasing slopes according to the changes in the hydraulic shielding factor.

Flow-current characteristics. A combination of linear dependencies of volume flow on the applied voltage with the non-linear current-voltage characteristics gives rise to non-linear flow-current characteristics, as shown in Fig. 12.

As is expected, increasing the inter-hole spacing makes the initial slope smaller (due to the stronger hydraulic shielding). However, it also makes the current-voltage characteristics more sub-linear (at positive voltages). In other words, the voltage needed to sustain a given current density is larger for more distant holes. According to the linear hydraulic problem, larger voltages produce proportionally larger volume flows. As a result, initially smaller flows increase dramatically within a certain current-density range so, at still larger currents, the flow rates at larger inter-hole spacings exceed the rates at smaller spacings.

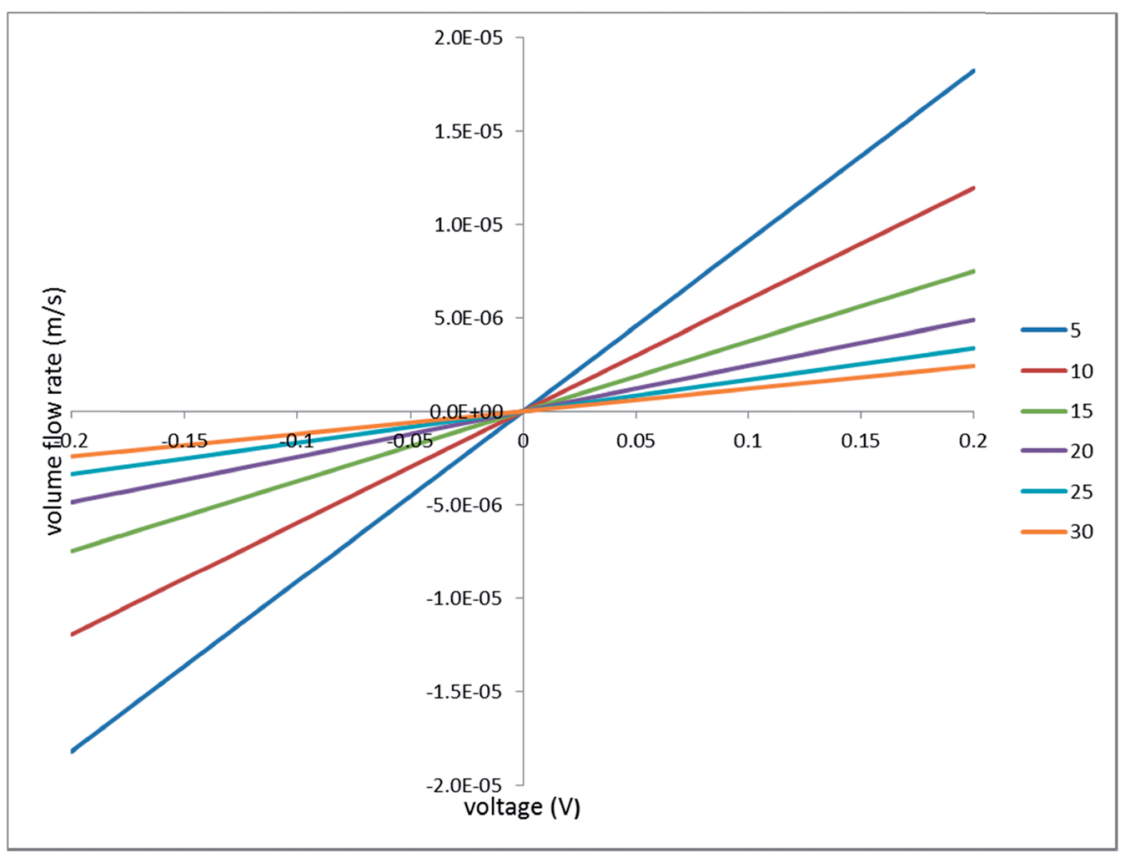

Fig. 11 Volume flux vs. applied voltage; the legend indicates the half-distance between the hole centers in micrometers. 


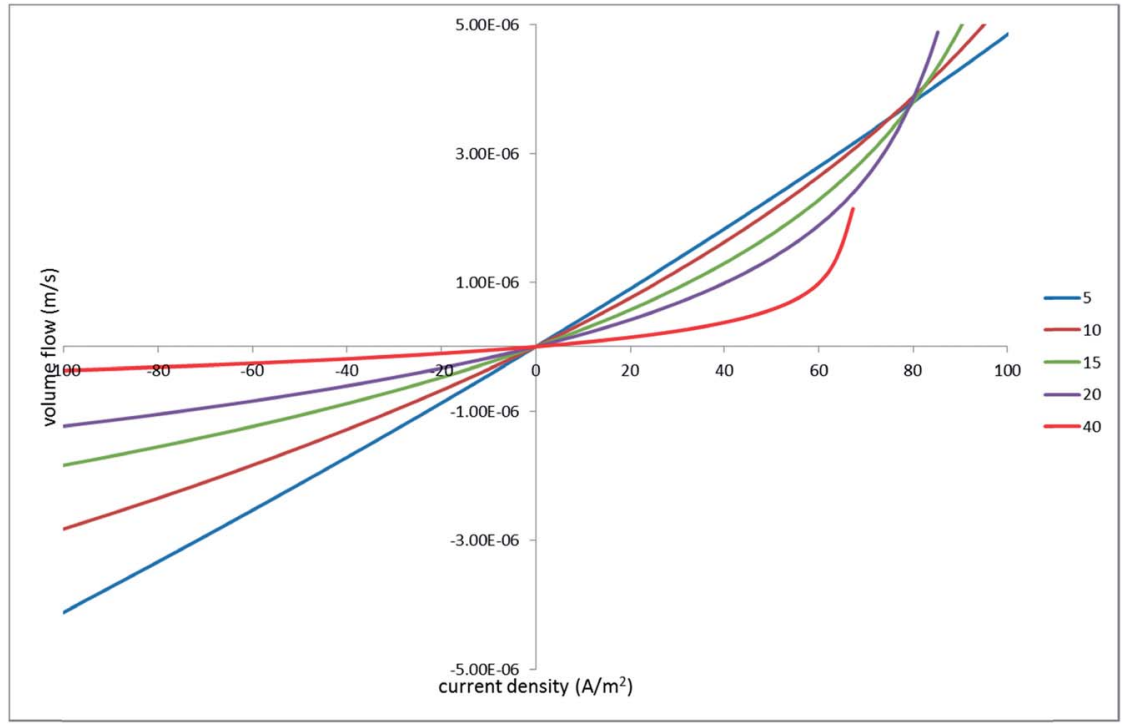

Fig. 12 Volume flux vs. current density; the legend indicates the half-distances between the hole centers in micrometers.

This feature is even more pronounced in terms of the net volume flow under ACEO conditions (see Fig. 13), which is defined as the sum of flows occurring at currents of the same magnitude but in opposite directions. In this case, the flows within the linear parts of the flow-current characteristics (at both voltage signs) cancel out and only the non-linear parts contribute.

Cycling current (rather than voltage) is relevant in view of its possible use in capacitive electrodes where the transferred charge must be equal to zero over the AC period. However, one should keep in mind that sustaining sufficiently high current densities at larger inter-hole spacings may require much too large voltages, that can give rise to undesired faradaic reactions at the electrodes. Actually, in all of the simulations shown in Fig. 13, the voltage-drop magnitude was limited by $300 \mathrm{mV}$. This is why the curves calculated for larger inter-hole spacings end at lower current densities.

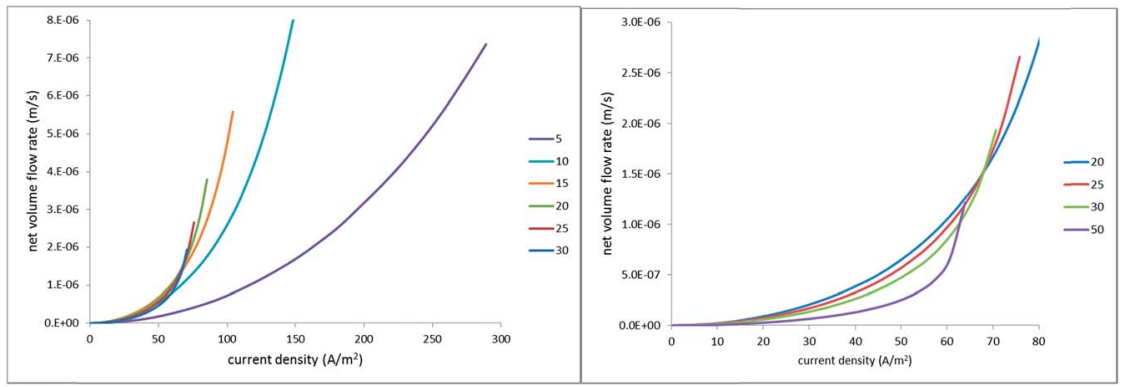

Fig. 13 Net EO flow rate vs. absolute value of current density; the legends indicate the half-distance between the hole centers in micrometers. 


\section{Conclusions}

We have explored theoretically a new configuration exhibiting non-linear and asymmetric electroosmosis due to ionic concentration polarization. This is achieved in a layered system where a (nano)porous medium is sandwiched (on one side) with a perforated ion-exchange membrane. The purpose of the perforations is to enable noticeable volume flow through the dense ion-exchange layer, which otherwise is almost hydraulically impermeable. The function of the ion-exchange layer itself is to produce current-induced concentration polarization up to rather high salinities.

The problem has been solved numerically for a model system with a square lattice of circular holes in the ion-exchange mask. For simplicity, the finite thickness of the mask was neglected and it was assumed to be ideally permselective in electrochemical terms. This analysis has confirmed that electroosmosis in such systems can be pronouncedly non-linear and asymmetric. The asymmetric EO gives rise to net EO flows over the period of AC when the current density is periodically cycled. We have also shown that the non-linearity and asymmetry get stronger with increasing inter-hole spacing and decreasing hole size. At the same time, making the holes more distant and smaller in size renders the EO flow smaller at lower current-densities. However, at larger currents it becomes more super-linear and this ultimately over-compensates the initially weaker flow. Notably, this occurs at larger voltage drops. In view of the low frequencies probably required for the concentration polarization to develop (and the EO asymmetry to occur in full), excessive voltages may lead to undesired electrode reactions. Therefore, in the context of practical applications, further analysis should include the electrodes (in view of the possible electrode reactions but also of the voltage drop on them). Further analysis also has to consider genuine non-stationary AC conditions. Nonetheless, even this simple steady-state analysis provides some initial guidance for the design of high-productivity and inexpensive AC electroosmotic pumps.

\section{References}

1 X. Wang, C. Cheng, S. Wang and S. Liu, Microfluid. Nanofluid., 2009, 6, 145162.

2 T. Eidsnes and O. Ellingsen, WO 1999000166 A1, Consensus As, 1998.

3 http://www.osmotex.ch/en/membrane/.

4 J. Wu, J. Appl. Phys., 2008, 103, 24907.

5 M. S. Yoon, B. J. Kim and H. J. Sung, Int. J. Heat Fluid Flow, 2008, 29, 269-280.

6 P. García-Sánchez and A. Ramos, Microfluid. Nanofluid., 2008, 5, 307-312.

7 M. Bazant and T. Squires, Phys. Rev. Lett., 2004, 92, 66101.

8 C.-T. Kuo and C.-H. Liu, Lab Chip, 2008, 8, 725.

9 P. Cervenka, J. Hrdlicka, M. Pribyl and D. Snita, IEEE Trans. Ind. Appl., 2010, 46, 1679-1691.

10 J. Hrdlicka, P. Cervenka, M. Pribyl and D. Snita, J. Appl. Electrochem., 2010, 40, 967-980.

11 T. A. Zangle, A. Mani and J. G. Santiago, Chem. Soc. Rev., 2010, 39, 1014-1035.

12 A. R. Kneller, D. G. Haywood and S. C. Jacobson, Anal. Chem., 2016, 88, 63906394. 
13 P. Jin, H. Mukaibo, L. P. Horne, G. W. Bishop and C. R. Martin, J. Am. Chem. Soc., 2010, 132, 2118-2119.

14 G. W. Bishop, M. M. Lopez, P. Ramiah Rajasekaran, X. Wu and C. R. Martin, J. Phys. Chem. C, 2015, 119, 16633-16638.

15 X. Wu, P. Ramiah Rajasekaran and C. R. Martin, ACS Nano, 2016, 10, 46374643.

16 A. Yaroshchuk, Microfluid. Nanofluid., 2012, 12, 615-624.

17 T. Xu and C. Huang, AIChE J., 2008, 54, 3147-3159.

18 R. J. Hunter, Zeta potential in colloid science: principles and applications, Academic Press, 1981, p. 386.

19 L. D. Landau and E. M. Lifshits, Electrodynamics of Continuous Media, Oxford University Press, 2nd edn, 1984. 